\title{
APPROXIMATE CROSS SECTIONS FOR OPTICALLY DISALLOWED EXCITATION OF ATOMS BY ELECTRONS
}

\author{
by L. VRIENS \\ Fysisch Laboratorium der Universiteit, Utrecht, Nederland
}

\section{Synopsis}

Formulae for approximate calculation of excitation cross sections for optically disallowed transitions are discussed. It is shown that the formula previously given by Drawin can be used in a different manner than pointed out by this author. This different manner seems to give reasonably accurate cross sections for small energies of the incident electrons, and gives the correct high energy limit. A comparison is made between approximate cross sections calculated here and already known Born cross sections.

Introduction. Knowledge of excitation and ionization cross sections of atoms is important in astrophysics and plasmaphysics. Experimentally determined cross sections are only available for a restricted number of transitions, in most cases only from the ground state. Theoretical calculations of cross sections are very complicated and have also been carried out only for a limited number of transitions. The results of such calculations are generally not very well in agreement with experiment for small energies of the impinging electrons. Approximate calculation of cross sections with simple empirical formulae often gives better results than complicated theoretical calculations. In previous papers we considered ionization and optically allowed excitation of atoms ${ }^{1}$ ). In this paper we consider optically disallowed excitation of atoms without exchange. Hence we restrict ourselves here to transitions such as $\mathrm{H} 1 \mathrm{~s} \rightarrow 2 \mathrm{~s}, 3 \mathrm{~s}, 4 \mathrm{~s}, 3 \mathrm{~d}, 5 \mathrm{f} ; \mathrm{H} 2 \mathrm{p} \rightarrow 3 \mathrm{p}, 6 \mathrm{f}$; He ${ }^{1} \mathrm{~S} \rightarrow 3^{1} \mathrm{~S}, 3^{1} \mathrm{D}, 4^{1} \mathrm{D}$; we neglect exchange in these transitions and do not consider transitions such as IIe $1^{1} \mathrm{~S} \rightarrow 2^{3} \mathrm{~S}, 3^{3} \mathrm{P}, 3^{3} \mathrm{D}$.

Empirical formulae. Scanlon and Milford ${ }^{2}$ ) have given empirical formulae for quadrupole excitation. These formulae can be used for approximate calculation of cross sections for small electron energies $E_{1}$, if the values of the cross section for two sufficiently large electron energies $E_{1}$ are known. Their formulae can also be used if the cross section for one sufficiently large electron energy $E_{1}$ and simultaneously the quadrupole matrix element are known. However, these authors pointed out themselves that 
for several transitions in atomic hydrogen their formulae give cross sections as large as two to six times the Born cross sections for small $E_{1}$. As it is much more probable that the Born approximation overestimates the cross sections for small $E_{1}$ than that it underestimates these cross sections (see for instance ref. 3), their empirical formulae do not seem to be very reliable.

Drawin ${ }^{4}$ ) has given the following empirical formula for optically disallowed excitation without exchange

$$
Q=Q_{m} \frac{4 U}{E_{1}}\left(1-\frac{U}{E_{1}}\right)
$$

where $U$ is the excitation energy and where he takes $Q_{m}$ equal to the maximum experimental cross section. In this manner, (1) can only be used if $Q_{m}$ is known experimentally. Absolute measurements of excitation cross sections in general are more difficult than relative measurements, which implies that if $Q_{m}$ is known absolutely, the excitation function is also known absolutely. Then application of (1) in the manner as pointed out by Drawin, is only useful a, to obtain an estimate of the $Q^{\prime}$ s for large $E_{1}, \mathrm{~b}$. for applications (e.g. if one wants to integrate over a Maxwellian electron energy distribution), c. to check the accuracy of (1). We note that theoretically calculated $Q_{m}$ values are not very reliable, so that use of them in (1) is not advisable.

The function (1) can also be used in a different manner, for which purpose we replace $4 Q_{m} U$ by $B$, so that

$$
Q=\frac{B}{E_{1}}\left(1-\frac{U}{E_{1}}\right)
$$

where $B$ is now chosen such that (2) gives the correct high energy limit ( $B$ is a constant for one transition; we note that our $B$ is not related to the Einstein absorption probability). Hence, for application of (2) only one cross section for a sufficiently large value of $E_{1}(\geqslant U)$ should be known; then the value of $B$ for that transition can be found immediately.

Approximate cross sections. For a number of transitions in atomic hydrogen, Born cross sections for large and for small $E_{1}$ are calculated by Scanlon and Milford ${ }^{2}$ ), and by McCoyd, Milford and Wahl $\left.{ }^{5}\right)$. With their $Q$ values for the largest $E_{1}$ values for which data are given $(\approx 1 \mathrm{keV})$, we calculated $B$ values, which for small $E_{1}$ were used for approximate calculation (eq. 2) of cross sections. In table I, the calculated $B$ values are listed. In table II, the approximately (eq. 2) calculated cross sections for small $E_{1}$ are compared with Born cross sections given by Scanlon e.a. and McCoyd e.a..

From table II it follows that for small $E_{1}$, (2) gives smaller cross sections than the Born approximation gives. As already pointed out, this is more 
TABLE I

\begin{tabular}{|c|c|c|c|c|c|}
\hline \multicolumn{7}{|c|}{ Calculated $B$ values for atomic hydrogen } \\
\hline trans. & $\begin{array}{c}B \\
\left(\pi a_{0}{ }^{2} \mathrm{eV}\right)\end{array}$ & trans. & $\begin{array}{c}B \\
\left(\pi a_{0}{ }^{2} \mathrm{eV}\right)\end{array}$ & trans. & $\begin{array}{c}B \\
\left(\pi a_{0}{ }^{2} \mathrm{eV}\right)\end{array}$ \\
\hline $1 \mathrm{~s} \rightarrow 2 \mathrm{~s}$ & 6.02 & $2 \mathrm{~s} \rightarrow 3 \mathrm{~d}$ & 228 & $3 \mathrm{p} \rightarrow 4 \mathrm{p}$ & 368 \\
$1 \mathrm{~s} \rightarrow 3 \mathrm{~s}$ & 1.20 & $2 \mathrm{p} \rightarrow 3 \mathrm{p}$ & 79 & $3 \mathrm{p} \rightarrow 4 \mathrm{f}$ & 717 \\
$2 \mathrm{~s} \rightarrow 4 \mathrm{~s}$ & 14.1 & $3 \mathrm{~s} \rightarrow 4 \mathrm{~s}$ & 311 & $3 \mathrm{~d} \rightarrow 4 \mathrm{~s}$ & 7.40 \\
$2 \mathrm{p} \rightarrow 4 \mathrm{f}$ & 21.1 & $3 \mathrm{~s} \rightarrow 4 \mathrm{~d}$ & 636 & $3 \mathrm{~d} \rightarrow 4 \mathrm{~d}$ & 295 \\
$2 \mathrm{~s} \rightarrow 3 \mathrm{~s}$ & 72.2 & $3 \mathrm{~s} \rightarrow 4 \mathrm{f}$ & 324 & & \\
\hline
\end{tabular}

TABLE II

\begin{tabular}{|c|c|c|c|c|c|c|c|c|c|}
\hline \multicolumn{10}{|c|}{$\begin{array}{c}\text { Comparison of Born cross sections }{ }^{2} \text { ) } \text { ) and cross sections calculated with (2); for disallowed } \\
\text { excitation of atomic hydrogen by electrons with energy } E_{1}\end{array}$} \\
\hline trans. & $\begin{array}{c}U \\
(\mathrm{eV})\end{array}$ & $\begin{array}{c}E_{1} \\
(\mathrm{eV})\end{array}$ & $\begin{array}{l}Q \text { Born } \\
\left(\pi a_{0}^{2}\right)\end{array}$ & $\begin{array}{c}Q \text { eq. (2) } \\
\left(\pi a_{0}{ }^{2}\right)\end{array}$ & trans & $\begin{array}{c}U \\
(\mathrm{eV})\end{array}$ & $\begin{array}{c}E_{1} \\
(\mathrm{eV})\end{array}$ & $\begin{array}{l}Q \text { Born } \\
\left(\pi a_{0}^{2}\right)\end{array}$ & $\begin{array}{l}Q \text { eq. (2) } \\
\left(\pi a_{0}^{2}\right)\end{array}$ \\
\hline \multirow[t]{3}{*}{$1 \mathrm{~s} \rightarrow 2 \mathrm{~s}$} & 10.196 & 13.34 & 0.246 & 0.106 & $3 s \rightarrow 4 s$ & 0.661 & 0.7391 & 64 & 45 \\
\hline & & 17.32 & 0.242 & 0.143 & & & 0.9675 & 116 & 102 \\
\hline & & 26.67 & 0.186 & 0.140 & & & 3.256 & 78 & 76 \\
\hline \multirow[t]{3}{*}{$1 s \rightarrow 3 s$} & 12.084 & 13.60 & 0.037 & 0.0098 & $3 s \rightarrow 4 d$ & 0.661 & 0.7391 & 120 & 91 \\
\hline & & 16.46 & 0.045 & 0.0193 & & & 0.9675 & 235 & 208 \\
\hline & & 19.82 & 0.044 & 0.0236 & & & 3.256 & 159 & 155 \\
\hline \multirow[t]{3}{*}{$2 s \rightarrow 4 s$} & 2.549 & 3.336 & 1.95 & 1.00 & $3 s \rightarrow 4 f$ & 0.661 & 0.7391 & 270 & 46 \\
\hline & & 4.331 & 2.04 & 1.34 & & & 0.9675 & 290 & 106 \\
\hline & & 10.77 & 1.14 & 1.00 & & & 3.256 & 99 & 80 \\
\hline \multirow[t]{3}{*}{$2 p \rightarrow 4 f$} & 2.549 & 2.604 & 0.58 & 0.17 & $3 p \rightarrow 4 p$ & 0.661 & 0.7391 & 83 & 53 \\
\hline & & 3.336 & 1.93 & 1.49 & & & 0.9675 & 145 & 120 \\
\hline & & 4.331 & 2.25 & 2.00 & & & 3.256 & 93 & 90 \\
\hline \multirow[t]{4}{*}{$2 s \rightarrow 3 s$} & 1.888 & 1.913 & 3.3 & 0.49 & $3 p \rightarrow 4 f$ & 0.661 & 0.7391 & 290 & 103 \\
\hline & & 2.604 & 12.4 & 7.6 & & & 0.9675 & 394 & 235 \\
\hline & & 3.401 & 12.7 & 9.4 & & & 3.256 & 193 & 175 \\
\hline & & 10.42 & 6.1 & 5.7 & $3 d \rightarrow 4 s$ & 0.661 & 0.7391 & 3.3 & 1.06 \\
\hline \multirow[t]{3}{*}{$2 s \rightarrow 3 d$} & 1.888 & 2.604 & 47 & 24 & & & 0.9675 & 4.22 & 2.43 \\
\hline & & 3.401 & 45 & 30 & & & 3.256 & 1.91 & 1.81 \\
\hline & & 10.42 & 19.8 & 18 & $3 d \rightarrow 4 d$ & 0.661 & 0.7391 & 100 & 42 \\
\hline \multirow[t]{4}{*}{$2 p \rightarrow 3 p$} & 1.888 & 1.913 & 4.2 & 0.54 & & & 0.9675 & 146 & 97 \\
\hline & & 2.604 & 14.9 & 8.3 & & & 3.256 & 78 & 73 \\
\hline & & 3.401 & 14.8 & 10.3 & & & & & \\
\hline & & 10.42 & 6.8 & 6.2 & & & & & \\
\hline
\end{tabular}

likely to be correct as the Born approximation in general overestimates the cross sections for small $E_{1}$. For some transitions the discrepancies between Born approximation and (2) are large for very small $E_{1}$ (near threshold). As the electron exchange process can give considerable contributions to the cross sections for very small $E_{1},(2)$ can underestimate the cross sections near threshold for some transitions. We should note that the Born approximation does not take account of exchange either. Further, Born approximation and (2) do not predict fine structure in the excitation functions near threshold as sometimes found in experiment 6 ). For completeness, we also have listed in table II $Q$ values for very small $E_{1}$. For $E_{1}>2 U$, we believe that (2) will be reasonably accurate. Equations (1) and (2) predict that the 
excitation functions reach a maximum for $E_{1}=2 U$. For several transitions in $\mathrm{He}$ this is very well in agreement with experiment ${ }^{6}$ ). For the transition $1 \mathrm{~s} \rightarrow 2 \mathrm{~s}$ in $\mathrm{H}$ the agreement is much less $\operatorname{good}^{3}$ ), but (2) still gives cross sections more in accordance with experiment than the Born approximation gives.

Note. For transitions in atomic hydrogen between states with principal quantum numbers $n=2$ and 3 , and between states with principal quantum numbers $n=3$ and 4 , the cross sections for optically allowed excitation increase $^{1}$ ) with increasing azimuthal quantum number $l$ of the initial state, while the cross sections for optically disallowed excitation decrease with increasing $l$. For ionization the situation is just the opposite ${ }^{1}$ ); for ionization from initial states with $n=2$ or 3 , the cross sections for optically allowed transitions are largest for small $l$ and the cross sections for optically disallowed transitions are largest for large $l$. In this sense, allowed and disallowed processes are competitive for these transitions. Ionization and excitation are also competitive; this also follows from the sum rules for the oscillator strengths ${ }^{7}$ ) and the generalized oscillator strengths ${ }^{8}$ ). It is questionable if this competitive behaviour is fully valid near threshold.

Acknowledgements. The author is indebted to Prof. Dr. J. A. Smit, Dr. J. M. Fluit and Prof. Dr R. Geballe for valuable comments on the manuscript of this paper and to G. J. H. Valkenburg for carrying out the calculations.

This work is part of the research programme of the "Stichting voor Fundamenteel Onderzoek der Materie" (F.O.M.) and was made possible by financial support from the "Nederlandse Organisatie voor Zuiver Wetenschappelijk Onderzoek" (Z.W.O.).

Received 1-4-65

\section{REFERENCES}

1) Vriens, L., Physica $\mathbf{3 1}$ (1965) 385 and $\mathbf{3 1}$ (1965) 1081.

2) Scanion, J. H. and Milford, S. N., Astrophys. J. 134 (1961) 724.

3) Seaton, M. J., Atomic and Molecular Processes, ed. D. R. Bates (Academic Press, New York, 1962) p. 407.

Taylor-Buzeman, A. J., thesis (Amsterdam, 1965) p. 21,

Stauffer, A. D. and McDowe11, M. R. C., Proc. Phys. Soc. 85 (1965) 61.

4) Drawin, H. W., Z. Naturforsch. 19 (1964) 1451.

5) McCoyd, G. C., Milford, S. N. and Wahl, J. J. Phys. Rev. 119 (1960) 149.

6) Smit, C., Heideman, H. G. M. and Smit, J. A., Physica 29 (1963) 245.

7) Dalgarno, A. and Lynn, N., Proc. Phys. Soc. A 70 (1957) 802 (see also ref. 1).

8) Mott, N. F. and Massey, H. S. W., The theory of atomic collisions, (Oxford, Clarendon press, 1949) p. 250. 